\title{
IMPACT OF NETWORK QUALITY DETERIORATION ON USER'S PERCEIVED OPERABILITY AND LIVE-MIGRATION OF VIRTUAL MACHINES IN REMOTE DESKTOP ENVIRONMENTS
}

\author{
Shin-ichi Kuribayashi \\ Department of Computer and Information Science, Seikei University, Japan
}

\begin{abstract}
The remote desktop environment (Virtual Desktop Infrastructure) is attracting interest as a way to strengthen security and support mobile access or telework. To realize the remote desktop environments, a remote desktop protocol is required to transfer information via a network about the user's operations made on the keyboard and mouse on a terminal to the remote server. The growing popularity of remote desktop environments makes it important to determine the factors that govern the user's perceived operability with a remote desktop protocol. It is also necessary important to find out the conditions for a wide-area live migration of virtual machines, to use resources efficiently in the remote desktop environments.
\end{abstract}

This paper examines the impact of network quality deterioration (long network delay, high packet loss, small downlink bandwidth) on a user's perceived operability in remote desktop environments, assuming RDP, PCoIP and ICA as remote desktop protocol. Next, this paper studies the impact of network quality on the performance of a live-migration of virtual machines in remote desktop environments.

\section{KEYWORDS}

Remote desktop environments, user's perceived operability, RDP, PCoIP, ICA

\section{INTRODUCTION}

A remote desktop environment (Virtual Desktop Infrastructure) is the practice of hosting a desktop operating system within a virtual machine (VM) running on a centralized server, which is one of the realization approaches of thin-client computing system. A remote desktop environment is attracting interest as a way to strengthen security, reduce the operation and management cost, and support mobile access or telework [1]-[8]. A remote desktop protocol is used in the desktop environment to transfer the user's operations on the keyboard and mouse of a terminal to the relevant server at a remote site. The server responds to the user's operations, by updating screen information and sending it back to the terminal.

A variety of remote desktop protocols have been proposed. Microsoft's Remote Desktop Protocol (RDP) [2], VMware's PC-over-IP (PCoIP)[3],[4] and Citrix's ICA/HDX [5] are the most commonly used. The performance of remote desktop protocols and the effect of network quality deterioration (long network delay, high packet loss rate, small downlink bandwidth) on the performance have been studied [8]-[17]. However, few studies have been made on the user's

DOI : $10.5121 /$ ijcnc.2014.6603 
perceived operability. The satisfaction of a client user would be heavily influenced by the quality of the network connection between the client and the server.

Another but related technology that is also attracting attention is a live migration of VMs to other servers over a wide area. The objectives are to use resources efficiently in order to reduce the operational cost, and to ensure service continuity in the event of a wide-area disaster [19]-[26]. A remote desktop environment can also be migrated over a wide area. Therefore, it is necessary to study the impacts of an increase in network delay and packet loss on the performance of the livemigration in remote desktop environments.

This paper examines the impact of network quality deterioration, such as long network delay, high packet loss and small downlink bandwidth, on a user's perceived operability in remote desktop environments, assuming the use of RDP, PCoIP and ICA which are representative remote desktop protocols. The main thrust of this paper is to study the relationship between network quality deterioration and the user's perceived operability, rather than the comparison of the three remote desktop protocols. The paper also evaluates the effect of an increase in network delay and packet loss on the performance of the live migration of VMs in remote desktop environments.

The rest of this paper is organized as follows. Section 2 explains related works. Section 3 examines the impact of network quality deterioration on a user's perceived operability in remote desktop environments, assuming RDP, PCoIP and RDP as remote desktop protocol. Section 4 clarifies the impact of network quality on the performance of a live-migration of virtual machines in remote desktop environments. Finally, Section 5 gives the conclusions. This paper is an extension of the study in Reference [28].

\section{RELATED WORK}

The performance of remote desktop protocols and the effect of network quality deterioration on the performance have been studied [8]-[17]. The satisfaction of a client user would be heavily influenced by the quality of the network connection between the client and the server. Reference [13] has evaluated the response time of a virtual remote desktop systems for different desktop applications (such as text editors, presentation creators and image processing tools), and has identified that the response time of more interactive applications is more sensitive to network delays. In Reference [14], the traffic caused by thin client based office applications is characterized and in Reference [15] the differences between several thin client architectures are analyzed. Reference [16] has proposed a toolkit to benchmark thin-client based virtual desktop environments, and has evaluated the performance of popular user applications, TCP/UDP based thin client protocols and remote user experience under a variety of system load and network health.

However, few studies considers the implications of a decreased network quality deterioration on user's perceived operability. Although References [17] has proposed to optimize the QoE of Citrix based Thin Client in WAN environments with speedscreen and input-buffer functions, it has not estimated the opinion score for user's perceived operability.

As the satisfaction of a client user would be heavily influenced by the quality of the network connection between the client and the server, it is necessary to examine in detail the impact of network quality deterioration (long network delay, high packet loss, small downlink bandwidth) on user's perceived operability. It is also required to evaluate the impact of remote desktop protocol to be used. This paper evaluates the user's perceived operability with a simplified rating scale method based on DMOS (Degradation Mean Opinion Score) [18], assuming three major remote desktop protocols, RDP, PCoIP and ICA. 
On the other hand, a live-migration is intended to move a virtual machine (VM) with virtually no disruption to the services being provided. There are studies that assume that VMs are moved over a wide area rather than keeping them confined in the same site [19]-[26]. Such wide-area migration will improve robustness against wide-area disasters and the effectiveness of load balancing. When a VM is moved to a distant center, the performance may deteriorate (e.g, slow response and decreased throughput), due to an increase in network delay or a reduction in bandwidths. Referenced [26] has proposed to automatically apply WAN accelerator to prevent degradation in performance when the network delay between the terminal and the center exceeds a certain threshold as a result of moving a VM. The live-migration over a wide area is also attractive for remote desktop environments, so as to use resources efficiently and to ensure service continuity in the event of a wide-area disaster. However, to the best of our knowledge, the performance of a live-migration in remote desktop environments has not been yet evaluated. This paper evaluates the impact of network quality on a live-migration of virtual machines in remote desktop environments.

\section{IMPACT OF NETWORK QUALITY DETERIORATION ON USER'S PERCEIVED OPERABILITY}

\subsection{Assumptions}

(1) Remote desktop protocols

Three representative remote desktop protocols, Microsoft RDP7.1, PCoIP (VMware View 5.0) and ICA 12 , are used.

(2) Evaluation environment

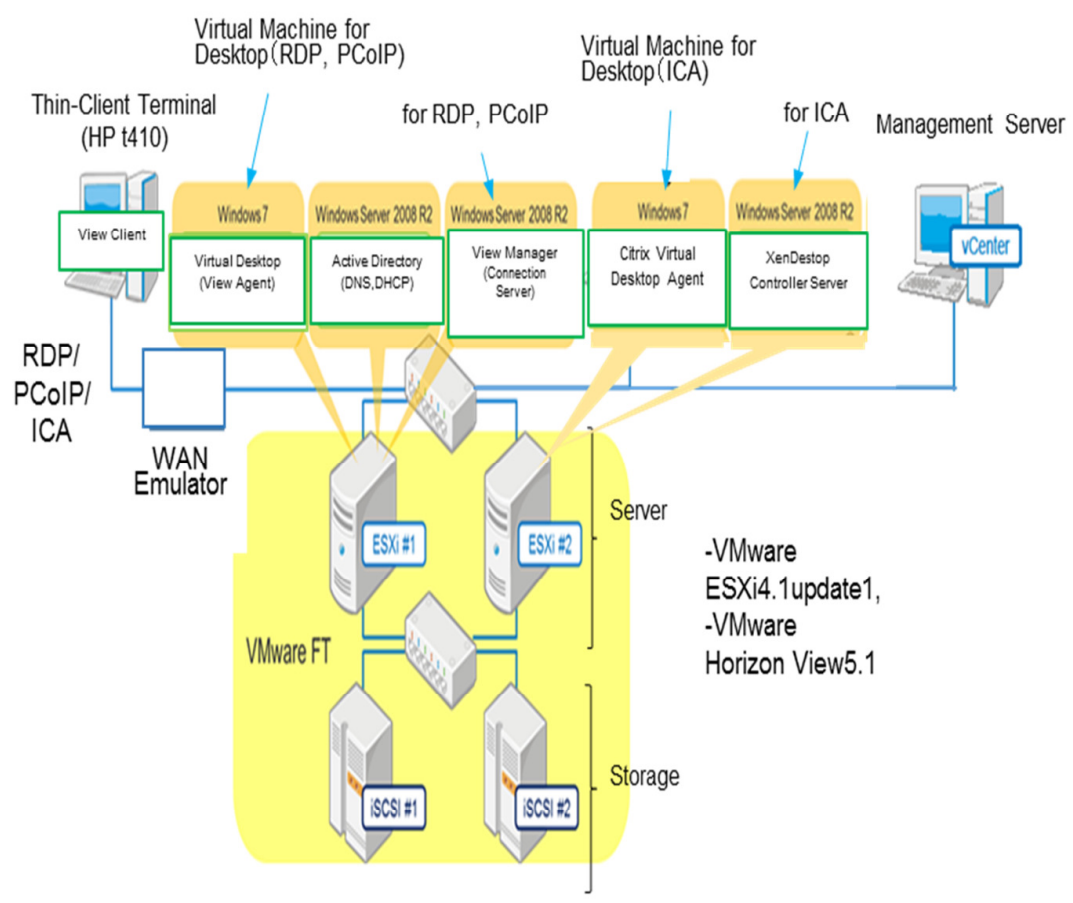

Figure1. System configuration for evaluation 
Figure 1 illustrates an overview of the system constructed for the evaluation. The virtual systems used is VMware ESXi4.1 update 1 and VMware Horizon View 5.1. Two VMs,Virtual Desktop (View Agent) VM and View Manager (Connection Server), are used for RDP and PCoIP. ICA is evaluated by constructing Citrix Virtual Desktop environment over VMware system, and two VMs, Citrix Virtual Desktop Agent and XenDesktop Controller Server, are used. Two physical servers (Proliant DL360 (Generation 7) from HP), two storage units (TS-RIX2.0TL/R5 from BUFFALO), and one thin client terminal (HPt410 Smart Zero Client from HP) are used. The speed of all physical links is 1 Gbps. A WAN emulator (Linktropy Mini2 from Apposite Technologies) is inserted between the server and the thin client terminal to generate network delay and packet loss, or to limit the downlink bandwidth. Windows 7 is used as the OS for the virtual machines that executes the processing needed for remote desktop. The user is able to select one of RDP, PCoIP and ICA on the operational window of the thin client terminal for access to virtual machines.

\subsection{Method of evaluating user's perceived operability}

The simplified rating scale method based on DMOS (Degradation Mean Opinion Score) [18] is used to evaluate the user's perceived operability with a remote desktop terminal. It uses fivegrade rating categories, and each category is assigned a score ranging from 1 to 5 . It is judged here that the user's perception is acceptable if the mean opinion score is 3.0 or higher.

\subsection{Evaluation method}

The following four different types of usage (or service profile) are used for evaluations:

$<$ Evaluation $1>$ Scrolling of a window of Notepad file

We enter a number from 1 to 1,000 , one number on each line, on a Windows 7 Notepad file. We scroll the window of this 1000-line file by continuing to press the down arrow.

$<$ Evaluation $2>$ Scrolling of a webpage

We create a 5000-line HTML file, with a number from 1 to 5000 written, one number on each line. Using Windows Internet Explorer 9, we scroll the window of the file by continuing to press the down arrow.

$<$ Evaluation 3> Automatic slideshow

Using OpenOffice 4.01 [27], we run a slideshow of a 21-slide file (slides change every 3 seconds).

$<$ Evaluation 4> Playback of a video

A video in WMV format with a video data rate of $1 \mathrm{Mbps}$ and an audio rate of $128 \mathrm{kbps}$ is used. The file size is $2.14 \mathrm{MB}$. Using Window Media Player 12, we play the video. 


\subsection{Evaluation results}

\subsubsection{Evaluation with RDP7.1}

(1) Impact of network delay and packet loss

Figure 2 shows how network delay and packet loss affect the mean opinion score of user's perceived operability for four Evaluations, explained in Section 3.3. The horizontal axis indicates network delay. The vertical axis shows the mean opinion score. The left bar in each figure indicates the mean opinion score in cases where there is no packet loss, and the right bar that in cases where the packet loss rate is $3 \%$.

\section{$<$ Evaluation 1>}

The perceived operability is not affected if the network delay is $500 \mathrm{~m} \mathrm{sec}$ or lower in cases where there is no packet loss, or if the network delay is $150 \mathrm{~m}$ sec or lower in cases where the packet loss rate is 3\%. The measured total scroll time is shown in Figure 3 for reference. It is clear that the network delay and packet loss rate do not affect the total scroll time so much. This means that it is difficult to judge the user's perceived operability with the scroll time. This was also true in the other types of evaluation.

$<$ Evaluation $2>$

The perceived operability is not affected if the network delay is $300 \mathrm{~m} \mathrm{sec}$ or lower in cases where there is no packet loss, or if the network delay is $200 \mathrm{~m} \mathrm{sec}$ or lower in cases where the packet loss rate is $3 \%$.

$<$ Evaluation 3>

The perceived operability is not affected if the network delay is $75 \mathrm{~m} \mathrm{sec}$ or lower in cases where there is no packet loss, or if the network delay is $25 \mathrm{~m} \mathrm{sec}$ or lower in case where the packet loss rate is $3 \%$.

$<$ Evaluation 4>

The perceived operability is affected unless there is no network delay and no packet loss. It is noted that the information transfer rate was about $20 \mathrm{Mbps}$. 

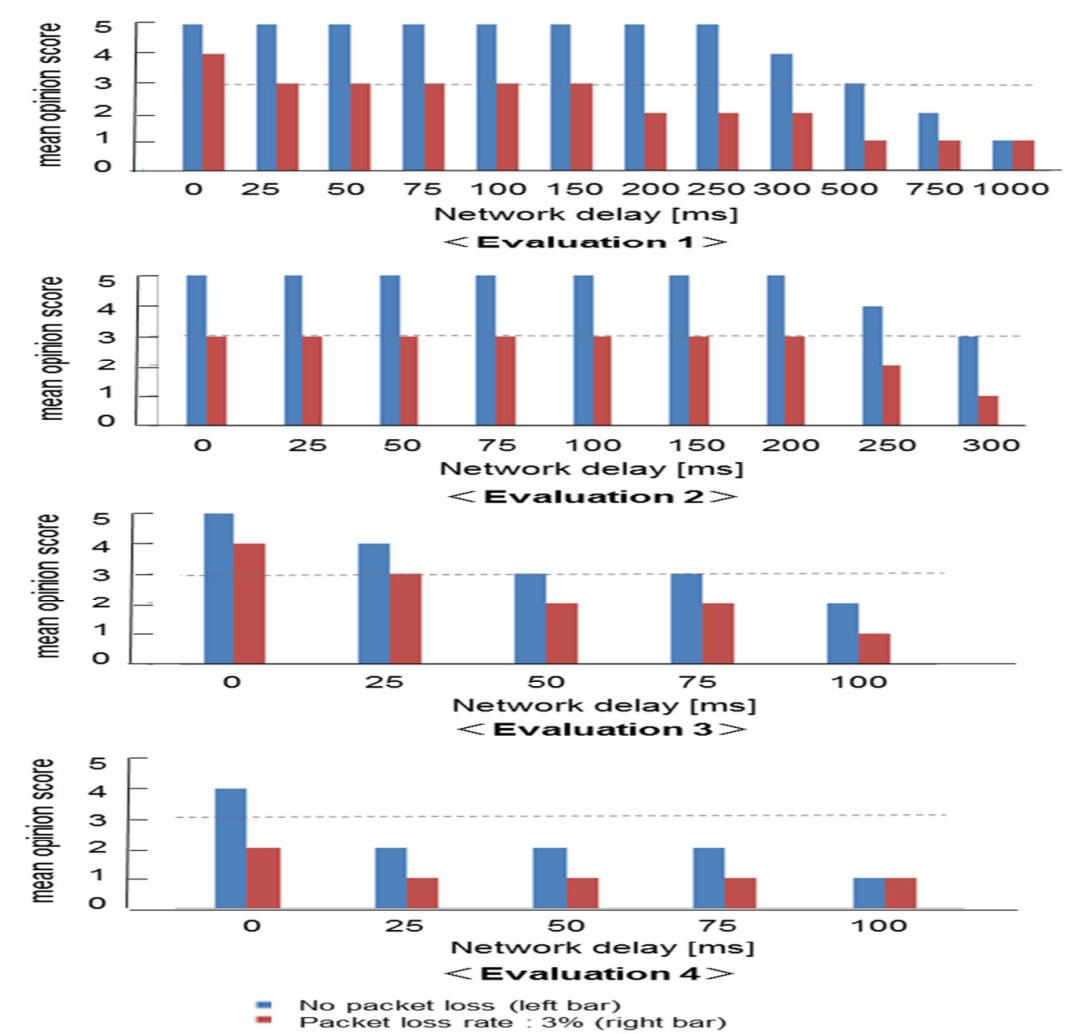

Figure 2. Evaluation of user's perceived operability with RDP7.1 (Network delay, Packet loss)

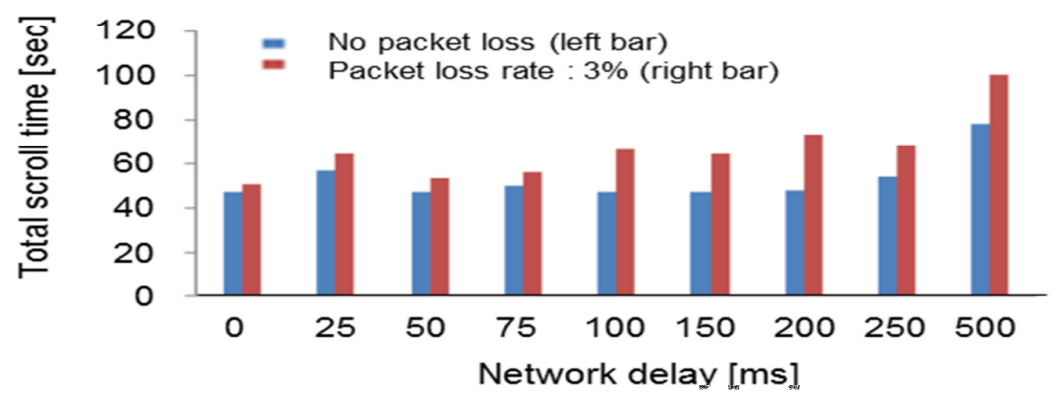

Figure 3. Total scroll time in RDP evaluation 1

(2) Impact of downlink bandwidth

Evaluations which would be easily affected by limiting downlink bandwidth are considered here. Figure 4 shows how downlink bandwidth affects the mean opinion score of user's perceived operability for Evaluations $3 \& 4$. The horizontal axis indicates downlink bandwidth. The vertical axis shows the mean opinion score. 
$<$ Evaluation 3>

The perceived operability is not affected if the downlink bandwidth is more than $300 \mathrm{k}$ bps, in cases where there is no packet loss and no packet delay.

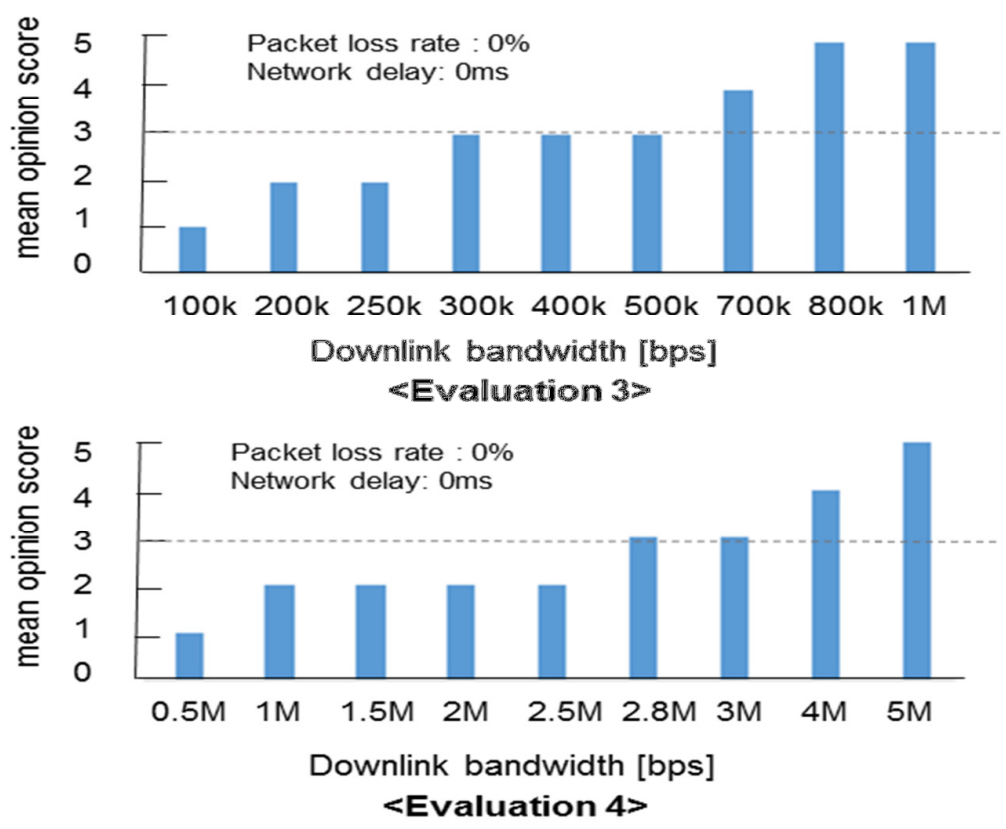

Figure 4. Evaluation of user's perceived operability with RDP7.1

(Limiting of downlink bandwidth)

$<$ Evaluation 4>

The perceived operability is not affected if the downlink bandwidth is more than $2.8 \mathrm{M}$ bps, in cases where there is no packet loss and no packet delay. 


\subsubsection{Evaluation with PCoIP}

(1) Impact of network delay and packet loss
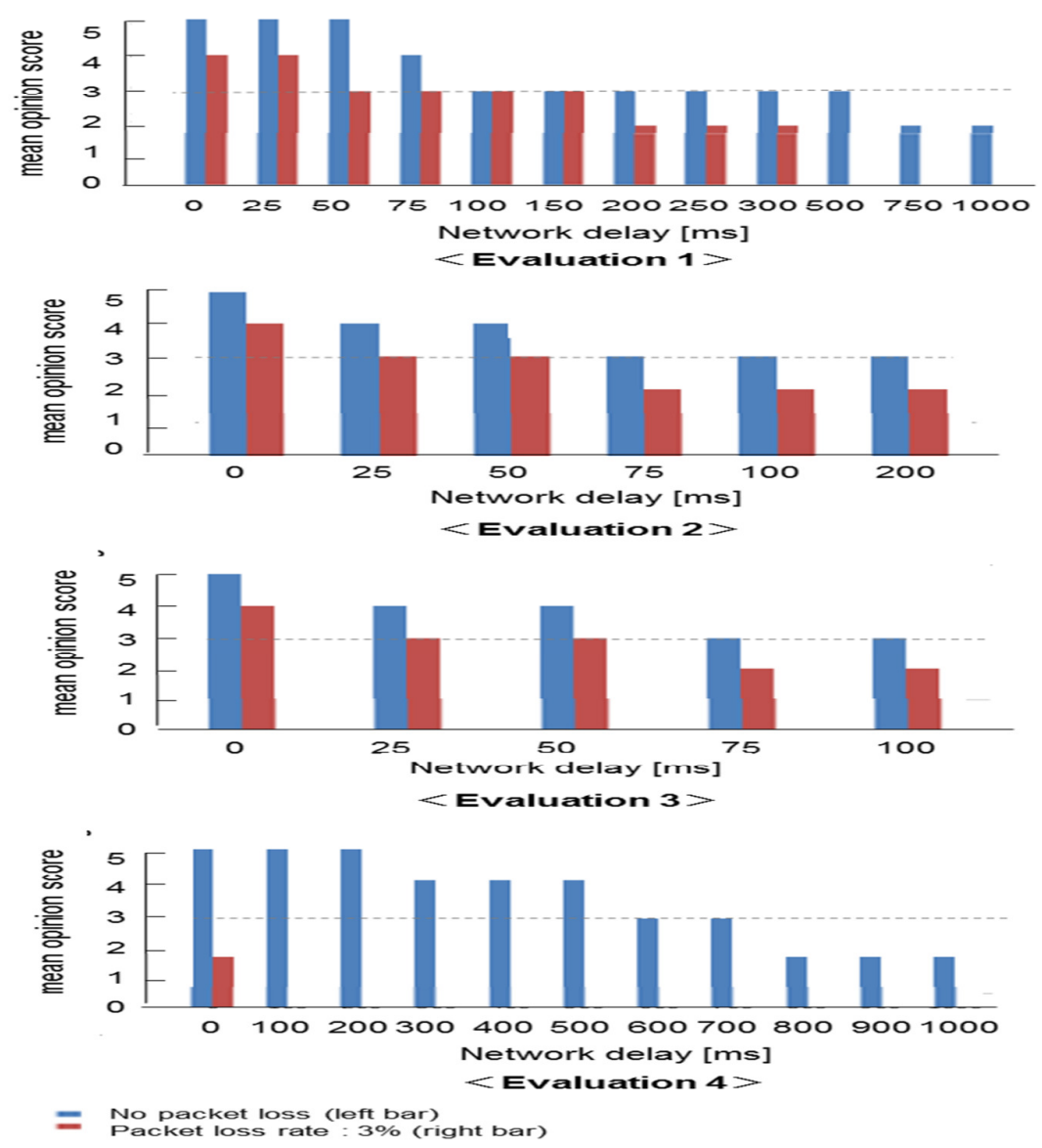

Figure 5. Evaluation of user's perceived operability with PColP (Network delay, Packet loss)

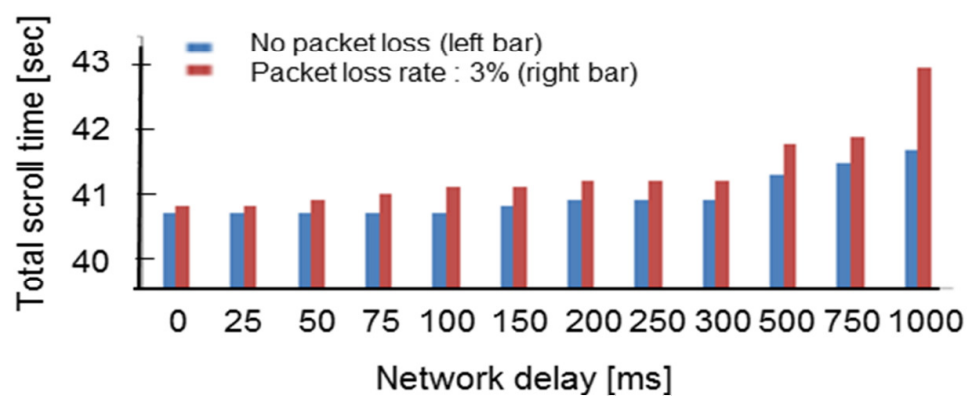

Figure 6. Total scroll time in PColP evaluation 1

As in Figure2, Figure 5 shows how an increase in network delay and packet loss rate affects the mean opinion score of user's perceived operability for four Evaluations. 
International Journal of Computer Networks \& Communications (IJCNC) Vol.6, No.6, November 2014

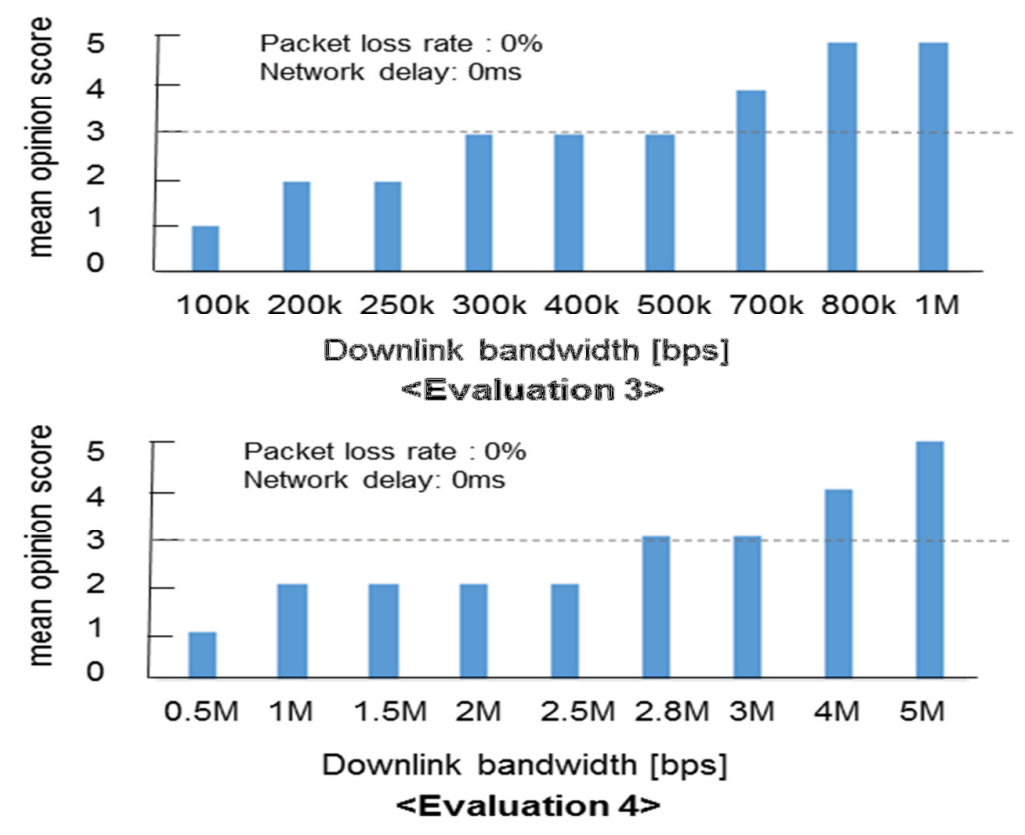

Figure 4. Evaluation of user's perceived operability with RDP7.1 (Limiting of downlink bandwidth)

$<$ Evaluation 1 >

The perceived operability is not affected if the network delay is $500 \mathrm{~m} \mathrm{sec}$ or lower in cases where there is no packet loss, or if the network delay is $150 \mathrm{~m} \mathrm{sec}$ or lower in cases where the packet loss rate is 3\%. The measured total scroll time is shown in Figure 6 for reference. As in Section 3.4.1(1), it is clear that the network delay and packet loss rate do not affect the total scroll time so much. This was also true in the other types of evaluation. 
$<$ Evaluation $2>$
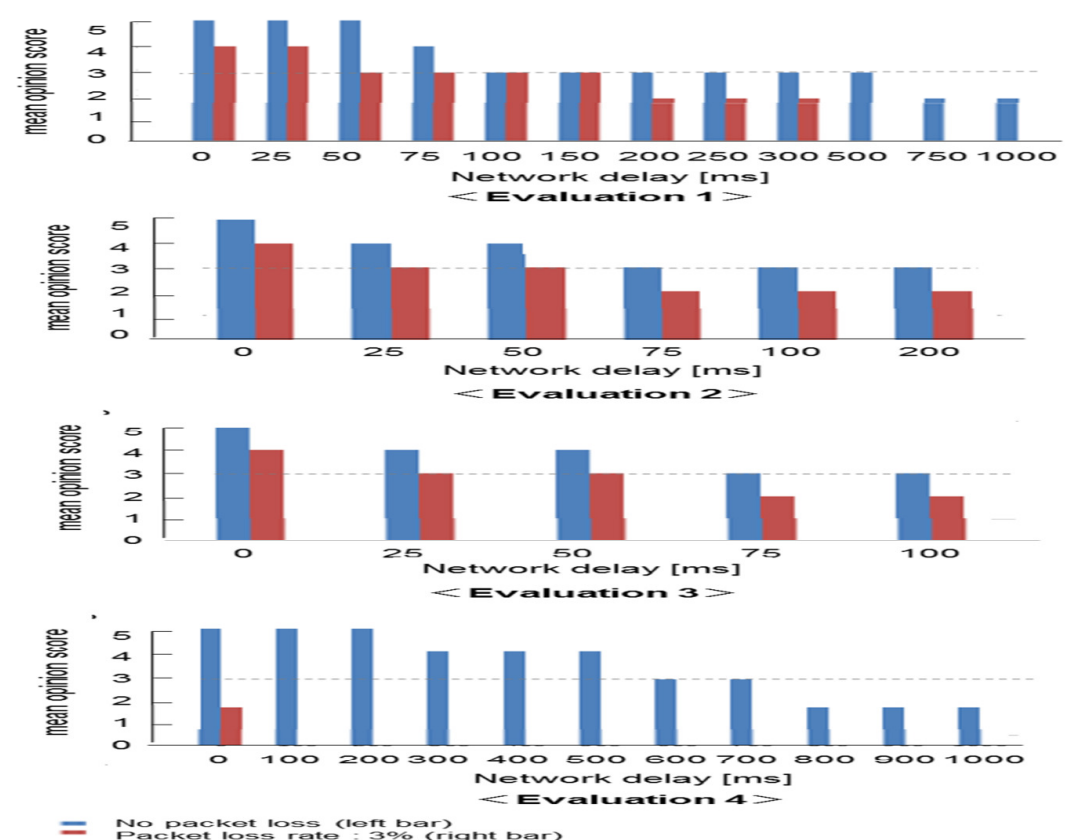

Figure 5. Evaluation of user's perceived operability with PColP (Network delay, Packet loss)

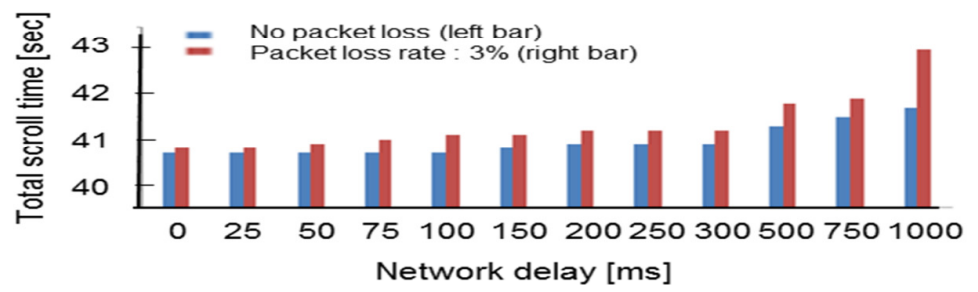

Figure 6. Total scroll time in PColP evaluation 1

The perceived operability is not affected if the network delay is $200 \mathrm{~m} \mathrm{sec}$ or lower in cases where there is no packet loss, or if the network delay is $50 \mathrm{~m} \mathrm{sec}$ or lower in cases where the packet loss rate is $3 \%$.

$<$ Evaluation $3>$

The perceived operability is not affected if the network delay is $100 \mathrm{~m}$ sec or lower in cases where there is no packet loss, or if the network delay is $50 \mathrm{~m} \mathrm{sec}$ or lower in cases where the packet loss rate is $3 \%$.

$<$ Evaluation $4>$

The perceived operability is not affected if the network delay is $700 \mathrm{~m} \mathrm{sec}$ or lower in cases where there is no packet loss. It is noted that the information transfer rate was about $5 \mathrm{Mbps}$, which was much lower than the cases where RDP was used. 


\section{(2) Impact of downlink bandwidth}

As in Figure4, Figure 7 shows how downlink bandwidth affects the mean opinion score of user's perceived operability for Evaluations $3 \& 4$. The horizontal axis indicates downlink bandwidth. The vertical axis shows the mean opinion score.

\section{$<$ Evaluation 3>}

The perceived operability is not affected if the downlink bandwidth is more than 50k bps, in cases where there is no packet loss and no packet delay.

$<$ Evaluation 4>

The perceived operability is not affected if the downlink bandwidth is more than $300 \mathrm{k}$ bps, in cases where there is no packet loss and no packet delay.

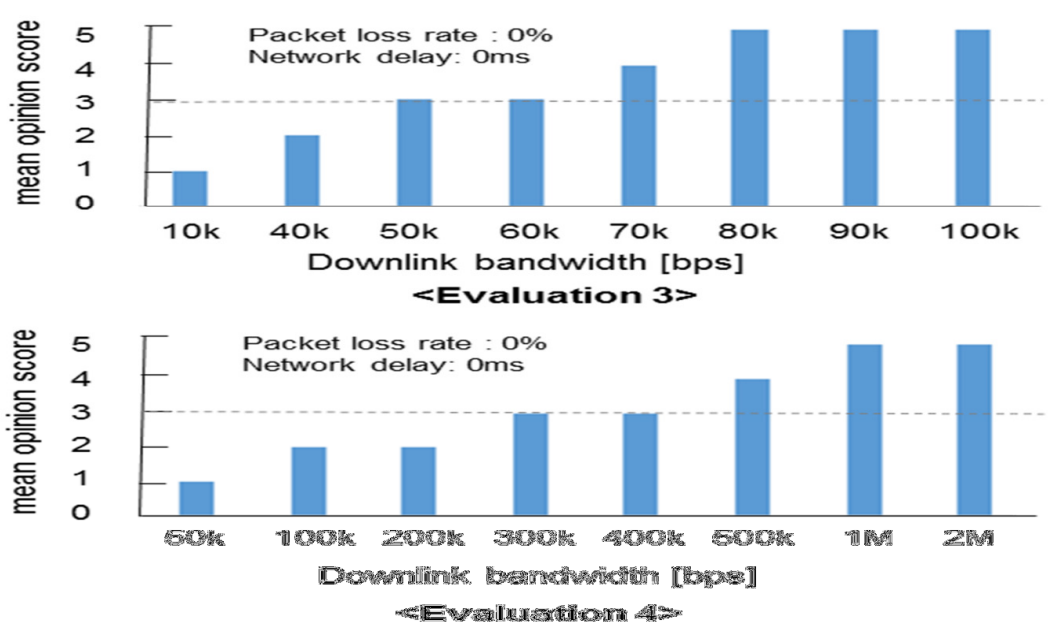

Figure 7. Evaluation of user's perceived operability with PColP (Limiting of downlink bandwidth)

\subsubsection{Evaluation with ICA12}

(1) Impact of network delay and packet loss

As in Figure 2, Figure 8 shows how an increase in network delay and packet loss rate affects the mean opinion score of user's perceived operability for three Evaluations.

$<$ Evaluation 1 $>$

The perceived operability is not affected if the network delay is $600 \mathrm{~m} \mathrm{sec}$ or lower in cases where there is no packet loss, or if the network delay is $300 \mathrm{~m} \mathrm{sec}$ or lower in cases where the packet loss rate is $3 \%$.

$<$ Evaluation $3>$

The perceived operability is not affected if the network delay is $3000 \mathrm{~m}$ sec or lower in cases where there is no packet loss and no network delay. 
$<$ Evaluation $4>$

The perceived operability is not affected if the network delay is $800 \mathrm{~m} \mathrm{sec}$ or lower in cases where there is no packet loss, or if the network delay is $100 \mathrm{~m} \mathrm{sec}$ or lower in cases where the packet loss rate is $3 \%$.

\section{(2) Impact of downlink bandwidth}

As in Figure4, Figure 9 shows how downlink bandwidth affects the mean opinion score of user's perceived operability for Evaluations $3 \& 4$. The horizontal axis indicates downlink bandwidth. The vertical axis shows the mean opinion score.

$<$ Evaluation 3>

The perceived operability is not affected if the downlink bandwidth is more than $4 \mathrm{k} \mathrm{bps}$, in cases where there is no packet loss and no packet delay.

$<$ Evaluation 4>

The perceived operability is not affected if the downlink bandwidth is more than $70 \mathrm{k}$ bps, in cases where there is no packet loss and no packet delay.

\subsubsection{Comparison of RDP, PCoIP and ICA}

A comparison of the evaluation results given in Sections 3.4.1, 3.4.2 and 3.4.3 indicates the following: When a remote desktop is used in the way in Evaluation 1 or 2, the perceived operability is less sensitive with RDP7.1. Conversely, the use of PCoIP and ICA12 makes the perceived operability less sensitive when a remote desktop is used in the way in Evaluation 3 or 4 . In particular, ICA12 makes the perceived operability less sensitive when a remote desktop is used in the way in Evaluation 4.

\section{IMPACT OF NETWORK QUALITY ON THE PERFORMANCE OF A LIVE-MIGRATION OF VMS}

The migration technology is used to move the memory spaces of VMs from one physical server to a different physical server while ensuring service continuity. A live-migration of VMs over a wide area have been studied as it can solve many problems such as load balancing and power saving. It could be also attractive for remote desktop environments, so as to use resources efficiently and to ensure service continuity in the event of a wide-area disaster. This section studies the impact of network quality on the performance of a live-migration of VMs in remote desktop environments, as in Figure 10

(1) Evaluation model

Except for the following changes, the same system shown in Figure 1 is used. RDP7. 1 is only applied for remote desktop protocol, and WAN emulator is moved between the physical servers to simulate network delay and packet loss. The physical speed of the link between the physical servers is $1 \mathrm{Gbps}$. 
The same usage in Evaluation 4 of Section 3.4.1 (playback of a video) is assumed and the time needed for live migration of a virtual machine executing the remote desktop processing with vMotion (live migration function of VMware) [3] is measured.
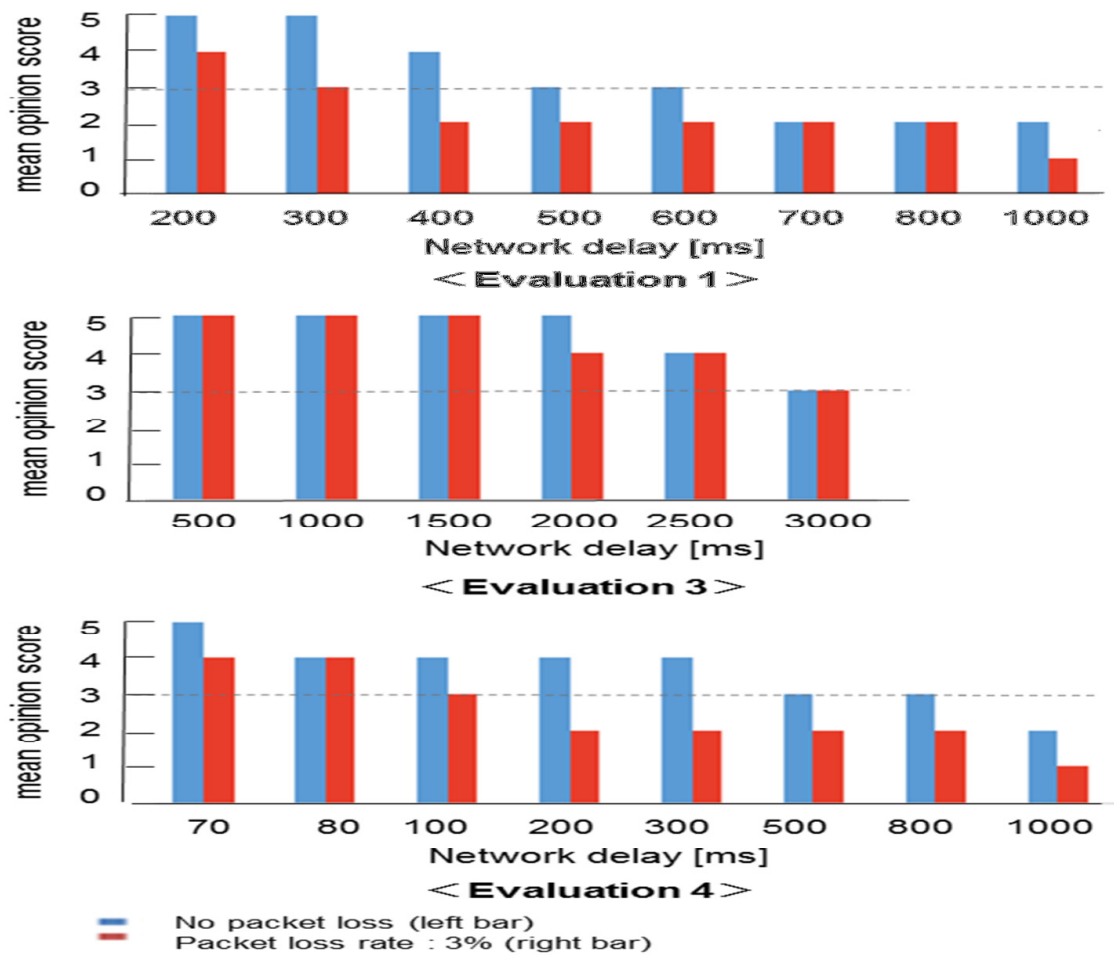

Figure 8. Evaluation of user's perceived operability with ICA12 (Network delay, Packet loss)

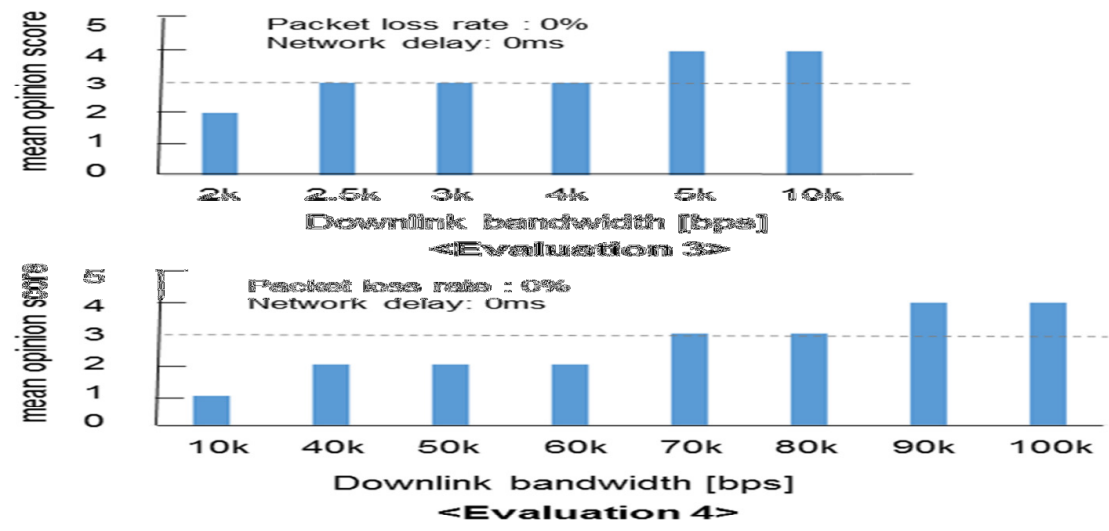

Figure 9. Evaluation of user's perceived operability with ICA12 (Limiting of downlink bandwidth) 


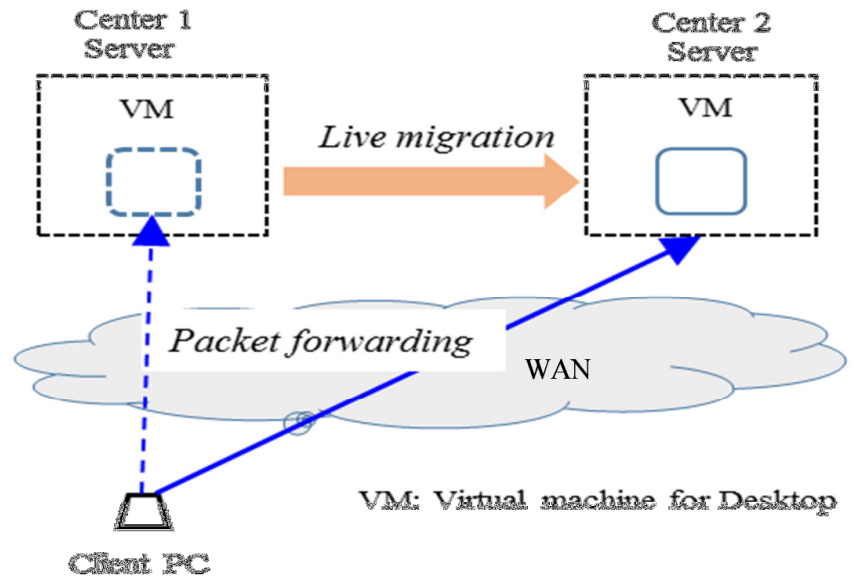

Figure 10. Image of wide-area live migration in remote desktop environments

(2) Evaluation results

Figure 11 shows how an increase in network delay and packet loss rate affects the total migration time. The horizontal axis indicates network delay or packet loss rate. The vertical axis shows the total migration time.

It is clear that the total migration time is about $20 \mathrm{sec}$ if there is no packet delay between the physical servers and no packet loss. To keep the total migration time within $80 \mathrm{sec}$, for example, the network delay must be $60 \mathrm{~m} \mathrm{sec}$ or lower (if there is no packet loss) or the packet loss rate must be less than $10 \%$ (if there is no network delay).

It was also measured that the migration was completed in about $40 \mathrm{sec}$ and $44 \mathrm{sec}$ with PCoIP and ICA respectively, when there was no network delay and no packet loss.

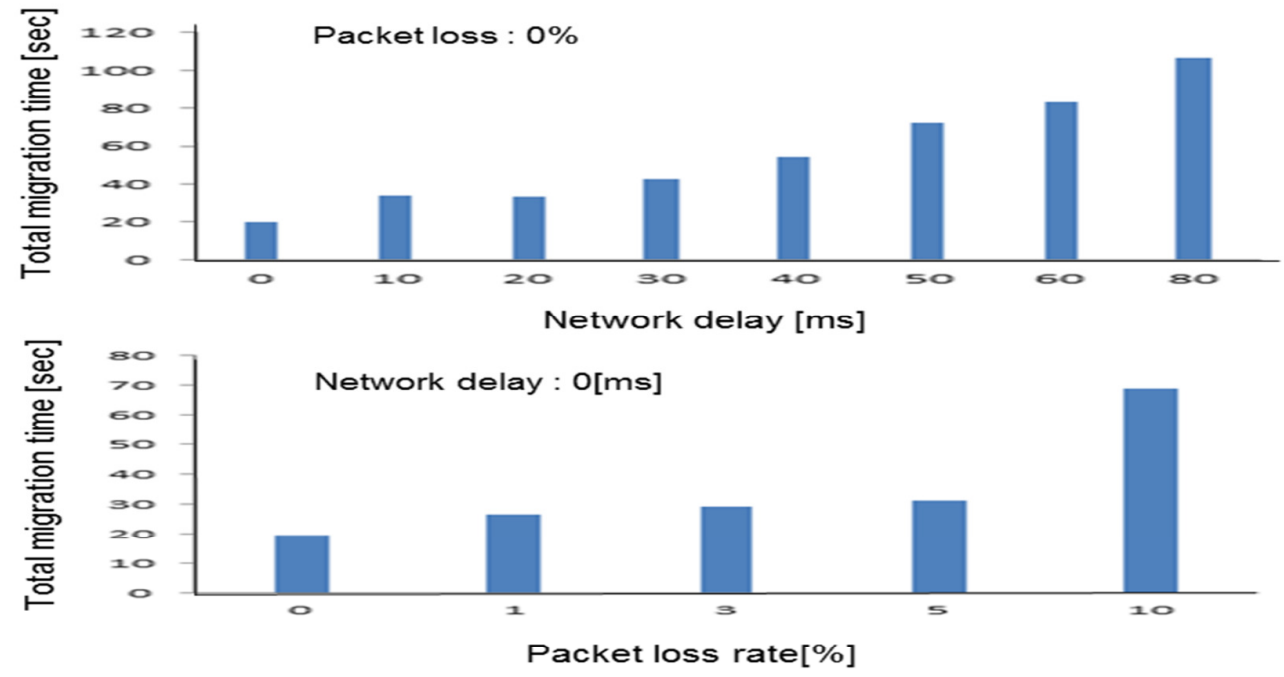

Figure 11. Total migration time with RDP7.1 


\section{CONCLUSIONS}

This paper has examined the impact of network quality deterioration on a user's perceived operability in remote desktop environments, assuming RDP, PCoIP and ICA as a remote desktop protocol. Although the scope of the evaluation in this paper is limited, it is found that, when RDP7.1 is used as a virtual desktop protocol, the user's perceived operability is acceptable when the user scrolls a webpage if the network delay is $200 \mathrm{~m} \mathrm{sec}$ in cases where the packet loss rate is $3 \%$. It is also found that, when PCoIP is used, the user's perceived operability is acceptable when playing a video, as long as the network delay is $700 \mathrm{~m} \mathrm{sec}$ or lower in cases where there is no packet loss. These results could be guidelines for the design and deployment of better remote desktop services.

This paper has also clarified the impact of network quality on the performance of a live-migration of virtual machines in remote desktop environments. It is clear by the evaluation that the network delay must be $60 \mathrm{~m} \mathrm{sec}$ or lower (in cases where there is no packet loss) or the packet loss rate must be less than $10 \%$ (in cases where there is no network delay), so as to keep the total migration time within $80 \mathrm{sec}$.

It is required to make more precise evaluation on various profiles and network quality. It is also required to evaluate with touch panel terminal as a remote desktop terminal in future.

\section{REFERENCES}

[1] R. Spruijt, "VDI Smackdown 2013 -solutions overview and feature comparison matrix-", May 2013.http://wp.techtarget.itmedia.co.jp/contents/?cid=13189

[2] Microsoft Corporation. Remote Desktop Protocol.http://msdn.microsoft.com/enus/library/cc240445.aspx

[3] VMware, "VMware View 5 with PCoIP" and "VMware Infrastructure Architecture Overview"http://www.vmware.com/files/pdf/view/VMware-View-5-PCoIP-Network-OptimizationGuide.pdf, http://www.vmware.com/pdf/vi_wp.pdf

[4] Teradici, "PC-over-IP Technology on enterprise networks", http://i.dell.com/sites/content/business/solutions/whitepapers/en/Documents/DRW__Teradici_Consid erations.pdf

[5] Citrix, "Ctrix HDX technologies"http://www.citrix.com/content/dam/citrix/en_us/documents/products-solutions/citrixhdx-technologies.pdf

[6] "SPICE”http://www.spice-space.org/

[7] “RealVNC”https://www.realvnc.com/

[8] S.J. Yang, J. Nieh, M. Selsky and N. Tiwari, “ The Performance of Remote Display Mechanisms for Thin-Client Computing", Proceedings of the 2002 USENIX Annual Technical Conference, June 2002.

[9] J. Nieh, S. Yang, and N. Novik, "Measuring Thin-Client Performance Using Slow-Motion benchmarking”, Proceedings of ACM Transactions on Computer Systems, Vol. 21, No. 1, Feb. 2003.

[10] The Tolly Group: MetaFrame XP Presentation Server Windows-based Application Access Performance and Functionality (2003)

[11] M. Lubonski, V. Gay and A. Simmonds, "An adaptation architecture to improve QoS of multimedia services for enterprise remote desktop protocols", Next Generation Internet Networks, April 2005.

[12] A. Lai and J. Nieh, "Limits of WideArea ThinClient Computing", Proceedings of the ACM SIGMETRICS 2002, June 15-19, 2002.

[13] N. Tolia, D. Andersen, and M. Satyanarayanan, "Quantifying Interactive User Experience on Thin Clients", in IEEE Computer Society Press Computer, vol. 39(3), 2006.

[14] Emmert, B., Binzenh"ofer, A., Schlosser, D., Weiß, M., "Source Traffic Characterization for Thin Client Based Office Applications",13th EUNICE, 2007. 
[15] Schlosser, D., Binzenh“ofer, A., Staehle, B., "Performance Comparison of Windowsbased Thin-Client Architectures", ATNAC'07, 2007.

[16] A. Berryman, P. Calyam, M. Honigford, and A.M. Lai, "VDBench: A Benchmarking Toolkit for Thin-client based Virtual Desktop Environments," 2nd IEEE International Conference on Cloud Computing Technology and Science, pp.480-487, Dec. 2010.

[17] D. Schlosser, B. Staehle, A. Binzenh"ofer, B. Boder, "Improving the QoE of Citrix Thin Client Users", IEEE ICC, 2010.

[18] ITU-T Recommendation P.800 http://www.itu.int/rec/T-REC-P.800-199608-I/en

[19] R. Bradford, E. Kotsovinos, A. Feldmann, and H. Schioberg: "Live wide-area migration of virtual machines including local persistent state", Proc. of 3rd international conference on Virtual Execution Environments, 2007.

[20] C. Clark, K. Fraser, S. Hand, J. G. Hansen, E. Jul, C. Limpach, I. Pratt and A. Warfield: "Live Migration of Virtual Machines", Proceedings of the 2nd USENIX Symposium on Networked Systems Design and Implementation, 2005.

[21] F. Travostino, P. Daspit, L. Gommans, C. Jog, J. Mambretti, I. Monga, B. Oudenaarde, S.Raghunath and P. Wang: "Seamless Live Migration of Virtual Machines over the MAN/WAN", Elsevier Future Generation Computer Systems, Vol.22, 2006.

[22] R. Bradford, E. Kotsovinos, A. Feldmann and H. Schioberg: "LiveWide-Area Migration of Virtual Machines Including Local Persistent State", VEE’07, June 2007.

[23] R. Bradford, E. Kotsovinos, A. Feldmann, and H. Schioberg: "Live wide-area migration of virtual machines including local persistent state", Proc. of 3rd international conference on Virtual Execution Environments, 2007.

[24] H. Liu, H. Jin, X. Liao, L. Hu and C. Yu: "Live Migration of Virtual Machine Based on Full System Trace and Replay", Proceedings of the 18th International Symposium on High Performance Distributed Computing (HPDC'09), June 2009.

[25] S. Akoush, R. Sohan, A. Rice, A. W. Moore and A. Hopper: "Predicting the Performance of Virtual Machine Migration", The 18th Annual IEEE/ACM International Symposium on Modeling, Analysis and Simulation of Computer and Telecommunication Systems (MASCOTS'10), Aug. 2010.

[26] S.Kuribayashi,"Improving Quality of Service and Reducing Power Consumption with WAN accelerator in Cloud Computing Environments", International journal of Computer Networks \& Communications (IJCNC), Vol.5, No.1, pp.41-52, Jan. 2013.

[27] OpenOffice http://www.openoffice.org/ja/

[28] R.Komatsu and S.Kuribayashi,"Impact of network quality deterioration on user's perceived operability in remote desktop environments", Proceeding of the 17-th International Conference on Network-Based Information Systems (NBiS-2014), pp.540-544,Sep. 2014. 